\section{Nedjeljka Balić-Nižić}

\section{Adolfo Mussafia e i suoi allievi zaratini}

Pregledni rad

Review article

UDK 821.131.1(497.5)

\section{Abstract}

Nell'intervento vengono presentati i lavori di alcuni rinomati scrittori e studiosi zaratini in lingua italiana della fine dell' 800 e dell'inizio del 900 che studiarono a Vienna sotto la guida del prof. Adolfo Mussafia (1835-1905), dedicandogli nel 1904, in occasione del suo centesimo semestre di insegnamento, un prezioso volume di testi letterari, studi e saggi, assieme ad altri allievi dalmati (Ad Adolfo Mussafia gli studenti italiani della Dalmazia. MDCCCLV-MCMIV, Spalato 1904). Partendo dalle ricerche dello studioso croato Žarko Muljačić, dedicate al suo illustre concittadino, si presentano tre contributi letterari e quattro saggi degli zaratini Cippico, Fabbrovich, Wondrich, Benevenia, Maddalena, Sabalich e Feoli, come specchio del carattere multidisciplinare del volume e come prova dell'influsso che il celebre professore ebbe sulla loro carriera, considerando che la maggior parte di loro dopo gli studi si dedicò all'attività di ricerca, ottenendo notevole successo nel campo giornalistico, letterario, storico e filologico.

Parole chiave: Adolfo Mussafia, scrittori zaratini in lingua italiana, relazioni letterarie italo-croate

Questo intervento ${ }^{1}$ è stato motivato dall'interesse che l'illustre romanista, professor Žarko Muljačić, fondatore del Dipartimento di Italianistica dell'Università di Zara e collaboratore, in varie forme e in varie fasi della sua fecondissima attività universitaria e filologica, di altre italianistiche croate tra cui anche quella di Pola, dimostrava per la vita e per l'opera del dalmata Adolfo Mussafia, ${ }^{2}$ il primo professore ordinario di filologia romanza all'Università di Vienna. Nei suoi studi dedicati all'illustre concittadino, il primo pubblicato nella rivista Građa i prilozi za povijest Dalmacije (MULAČıć 1997) e I'altro in Estudis Romànics (MULAČıć 2002), il rinomato romanista croato riporta interessanti dati biografici sul Mussafia, risolvendone l'enigma dell'anno

${ }^{1}$ L'intervento è stato presentato al Convegno internazionale in onore del Prof. Žarko Muljačić (1922 2009), organizzato dal Dipartimento di Italianistica della Facoltà di Lettere e Filosofia dell'Università di Zagabria, 15-17 novembre 2012.

${ }^{2}$ Nato a Spalato, il 15 febbraio 1835, morto a Firenze, il 9 giugno 1905. di nascita. Vari studiosi credevano, infatti, che Mussafia, uno dei dieci linguist provenienti dal territorio dell'odierna Croazia annoverati tra i più eminenti linguisti del mondo, ${ }^{3}$ fosse nato nel 1834 . Muljačić invece sostiene che il grande filologo fosse nato nel 1835 , spiegando che l'errore fu causato da un ritardato uso del "calendario veneziano" (more veneto), secondo il quale l'anno iniziava il primo marzo. Oltre a chiarire la data di nascita, Muljačić, nell'articolo sopraccitato riporta altri dati sul suo concittadino: sul suo vero nome (Abraham, Arturo, Abram, detto Adolfo), sui membri della famiglia, e in particolare sul padre (Johannes Amadeus; Jakob /figlio di/ Amedeo) Cerca, inoltre, di spiegare il plurilinguismo mussafiano che comprendeva la buona conoscenza di ebreo-spagnolo, italiano-veneto, però non afferma con sicurezza la conoscenza del croato, né del tedesco, quest'ultimo imparato molto probabilmente dopo I'arrivo a Vienna. Oltre alle lingue menzionate, sembra che il Mussafia nel corso della proficua carriera universitaria potesse leggere anche i testi in inglese, ceco, olandese, svedese, russo e polacco. Muljačić in seguito riporta dati sugli studi di medicina (in)compiuti dal Mussafia, iniziati secondo il desiderio dei genitori e abbandonati dopo 4 semestri. Come fatto particolarmente interessante si menziona il breve periodo in cui Mussafia apprese il tedesco, cominciando molto presto a pubblicare articoli e studi in questa lingua. Già nel 1860 uscì a Vienna la prima edizione della sua famosa grammatica italiana per i tedeschi, Italienische Sprachlehre (MUSSAFIA 1860) diventata, per le sue numerose ristampe e rifacimenti che uscivano fino alla metà del ' 900 , un termine (der Mussafia). Muljačić mette in rilievo le curiosità della carriera universitaria del Mussafia sviluppatasi in strane circostanze: in un periodo relativamente breve è diventato professore associato (1860), professore associato confermato (1863), e professore ordinario (1867), benché non avesse mai studiato né la filologia romanza, né nessun'altra filologia. La conoscenza necessaria l'ha acquistata leggendo, in particolare le opere del fondatore della filologia romanza, il professor Friedrich Christian Diez di Bonn (17941876), ${ }^{4}$ e frequentando le lezioni alla Facoltà di Lettere di Vienna, dove faceva tirocinio ad alcune lezioni ed esercizi di lingua italiana e alle cosiddette Lecturae Dantis. La carriera di Mussafia, secondo le fonti (Mu」Ačić 2002; TOLomeo 2012), ebbe un modesto inizio, dato che, rimasto senza la borsa di studio del Comune ebreo di Spalato (ottenuta dopo la morte del padre) a causa degli studi di medicina interrotti, doveva arrangiarsi in var modi. Un'occasione propizia si presentò con il tentativo del ministero dell'istruzione austriaco di risolvere problemi con i laureati in diverse altre materie che volevano insegnare nei licei con lingua d'insegnamento italiana in varie parti dell'Impero (tra

${ }^{3}$ Altri rinomati linguisti che fanno parte di questo gruppo sono: Henrik Barić (1888-1957), Matteo Giulio Bartoli (1873-1946), Pier Gabriele Goidànich (1868-1953), Josip Hamm (1905-1986), Stjepan Ivšić (1884-1962), Tomislav Maretić (1854-1938), Milan Rešetar (1860-19429, Petar Skok (18811956) e Niccolò Tommaseo (1802-1874).

${ }^{4}$ Tra le sue opere la più importante è la Grammatik der romanischen Sprachen, I-III, Bonn, 1836-1843, pietra miliare di quella nuova disciplina. 
I'altro, numerosi licei in Lombardia e Veneto). Così ad Adolfo Mussafia fu offerto di lavorare gratis come secondo maestro di lingua e letteratura italiana per gli studenti tedeschi e italiani che avevano l'intenzione di lavorare nei licei con lingua d'insegnamento italiana. Oltre a ciò, il giovane spalatino presto trovò anche un lavoro pagato, in Hofbibliothek, cioè nella Biblioteca della Corte. Dopo scrisse numerosi saggi e studi, divenne membro corrispondente dell'Accademia delle Scienze a Vienna e membro della presidenza della Società dantologica (Deutsche Dante-Gesellschaft, 1865), partecipando in varie iniziative e attività, tra l'altro nell'organizzazione per la celebrazione del centenario dantesco. Come bibliotecario ha aiutato molti colleghi, a.e. il veneziano Emilio Teza, il collega degli studi universitari, che in segno di gratitudine gli ha inviato la sua traduzione di un'ode del poeta francese J.M. de Hérédia (II Niagara), con dedica: "Ad Adolfo Mussafia, compiuto l'anno di lui sessantesimo... oggi XV, febbr. MDCCCXCV" (MuLAČić 1997). Alla fine ha ottenuto il Dr.h.c. (Doctor honoris causa), perché, per dirla con Muljačić, era un po' "imbarazzante" che i dottorandi fossero esaminati da un professore che non aveva il dottorato. Nonostante il successo ottenuto a Vienna, Mussafia cercava invano un lavoro in Italia che verso la fine della vita visitava spesso per ragioni di salute, spostandovisi definitivamente nell'ottobre 1904 e scegliendo Firenze come luogo di dimora. Per il suo compleanno nel 1905, pochi mesi prima della morte, alcuni suoi rinomati colleghi (Isidoro Del Lungo, Guido Mazzoni, E.G. Parodi e Pio Rajna) gli consegnarono un volume di lusso degli Atti pubblicati in Germania in suo onore (Bausteine zur Romanische Philologie für Adolfo Mussafia zum 15. Februar 1905).

Alla fine dell'esauriente studio su Mussafia, Muljačić dà suggerimenti per le future ricerche sull'insigne professore. Accennando alla sua voluminosa corrispondenza, parzialmente pubblicata, tra i compiti proposti a futuri ricercatori c’è anche quello di esaminare il rapporto tra il Mussafia e i suoi ex allievi croati, in particolare con Petar Skok.

Da uno dei capitoli di questo studio, intitolato "Onorificenze", si evince che Mussafia fu molto apprezzato sia dai colleghi professori e studiosi, sia dai suoi allievi dalmati, con cui era in stretti rapporti professionali e di amicizia, seguendo con attenzione la loro attività e appoggiandoli anche dopo gli studi. Non meraviglia quindi che tra le numerose onorificenze ci fosse anche il volume intitolato Ad Adolfo Mussafia gli studenti italiani della Dalmazia. MDCCCLV-MCMIV, (Spalato, 1904) scritto in occasione del suo centesimo semestre di lezioni e consegnatogli a Firenze dove trascorse gli ultimi mesi della vita.

Nel volume composto di 240 pagine, edito per cura della Società degli studenti italiani della Dalmazia si trovano contributi di: Giovanni Devich, decano capitolare di Spalato, che scrive Su I'lllyricum Sacrum di Daniele Farlati; Paolo Mazzoleni di Sebenico, il noto studioso degli scritti tommaseiani tra cui, per quell'occasione, pubblica un discorso inedito sui Diritti degli Israeliti alla civile eguaglianza; Giacomo Marcocchia di
Spalato, insegnante e studioso di storia con un approfondito studio sul Principio informatore del dolce stil nuovo; Carmelo Scrivanich di Sagrado nel Friuli orientale, con il trattato Una deduzione dalla teoria atomica, ed Alessandro Dudan di Spalato, che vi fornisce un'apposita nota. Oltre a questi, ci sono ancora sette contributi firmati da autori zaratini che volevano rendere omaggio all'illustre professore (Antonio Cippico, Giuseppe Fabbrovich, Giorgio Wondrich, Lorenzo Benevenia, Edgardo Maddalena, Giuseppe Sabalich e Gaetano Feoli)..$^{5}$ Dei loro sette contributi, che saranno brevemente presentati in seguito, tre sono testi letterari e quattro trattati storico-letterari e/o linguistici.

Antonio Cippico (1877-1935), nato a Zara, ma di origini traurine, è scrittore, professore di lingua e letteratura italiana in Inghilterra, nel periodo tra le due guerre mondiali anche politico e membro del Senato Italiano. Nel volume mussafiano pubblica la canzone Alla Dalmazia (CIPPICO 1904: 45-47) in cui, in maniera tommaseiana, esprime da una parte l'ammirazione per la bellezza della provincia nativa, soprattutto per la sua resistenza storica nei confronti di diversi invasori, mentre dall'altra parte si lamenta dell'attuale situazione in cui è minacciata la sua particolare posizione, facendo riferimento al pericolo della germanizzazione o slavizzazione della provincia e al fatto che si trova in balia di diversi interessi:

\section{O Dalmazia, indoma}

contra il Magiaro e l'Osmano,

contra Venezia e Roma

e contra a'l croatico bano;

o Dalmazia, o Madre

proterva ed oltre i secoli ribelle,

ben ti sovvenga, or sei vinta ed imbelle!

ché più barbare squadre,

i figli tuoi, coorte obliviosa,

oggi te mercan cui piace e dispiace!

(CIPPICO 2004: 46)

Gli ultimi versi della strofa citata fanno ricordare le simili riflessioni del grande Tommaseo espresse nella sua famosa poesia Alla Dalmazia:

Spregio o pietate alle superbe genti,

O poveretta mia, suona il tuo nome.

Siccome il braccio che, da corpo vivo,

Mezzo reciso, dolorosa noia,

Spenzola, in te così la vita altrui

Scarsa, o Dalmazia, e con dolor s'infonde.

Serbica e Turca ed Itala e Francese,

${ }^{5}$ Va detto che l'elenco di allievi zaratini di Mussafia è assai più lungo e contiene altri nomi rinomat quali Riccardo Forster, scrittore, giornalista e critico; Vitaliano Brunelli, storico e dantista; Angelo Nani, letterato ed editore; Carlo Brozovich, Matteo Fradelich, Biagio Costa e numerosi altri. 
Nè ben d'altrui né tua ben fosti mai:

Patria viva non hai chi di te nacque.

(TOMMASEO 1872: 31)

Cippico nella sua poesia accentua la particolare posizione e il ruolo secolare della Dalmazia di fare da collegamento, da ponte tra l'Oriente e Occidente. Nella ripetizione delle invocazioni "O mia Patria, o mia terra" si rivela il nesso intertestuale con il Va pensiero verdiano, che oggi, dopo un secolo di risvolte nell'assesto politico europeo, sembra quasi una profezia della patria dalmata che qualche decennio dopo sarebbe stata perduta. Bisogna sottolineare che Cippico, noto per il suo orientamento pro-italiano, offre un'immagine della Dalmazia sola, non appartenente a nessuna parte, ma, nuovamente sotto l'influsso delle idee tommaseiane, funzionante da ponte tra le due civiltà che vi coesistono da secoli (fra le slave e le genti latine):

\section{O mia Patria, rapace}

l'orda nuova s'avventa

in tua spoglia cruenta!

O mia terra, ma spenta

non tutta è la virtù che ti fe bella:

ne' I tuo Cuore d'ancella

arde la fiamma eterna,

che a' tuoi fati governa.

Sola e grande su 'I mare,

fra le slave e le genti latine,

Tu, ponte primevo ed altare,

$[\ldots]$

(CIPPICO 1904: 46-47)

Giuseppe Fabbrovich, zaratino di nascita che molto presto si trasferisce in Italia (Cormons nel Friuli orientale), e della cui attività letteraria si ricavano dati soprattutto grazie alle notizie riportate in quegli anni dal giornale cittadino // Dalmata che informa di un suo volume di poesie Sogni uscito nel 1904 a Milano coi tipi di Treves (BALIć-Nižlć 2008: 157), in questo volume, dedicato ad Adolfo Mussafia, pubblica alcuni brani del suo romanzo Vittime (Frammenti d'un romanzo... allo studio) (FABBROVICH 1904: 49-77). Le protagoniste, tre donne innamorate dello stesso uomo, sono le vinte, che, come riportato nel titolo stesso, diventano vittime dei propri sentimenti e dei rapporti colpevoli. Il carattere di ognuna delle donne, e il tipo di rapporto con Giuliano, l'uomo fatale e "lo spirito demoniaco", viene illustrato, oltre che dalla descrizione dell'aspetto fisico, anche dall'ambiente, cioè dallo scenario in cui "cadono" sopraffatte dai sentimenti e dalle sensazioni che le inducono nel peccato: il paesaggio alpino, una città al mare non definita in contrasto con un villaggio vicino alle miniere appena saltate in aria.
Giorgio Wondrich, impiegato del ministero delle Finanze presso gli uffici zaratini, spostatosi dopo a Trieste, dove fu redattore della rivista letteraria II Palvese, è noto anche come autore di componimenti poetici d'occasione, di saggi critici e di opere teatrali (BALIĆ-NIžıć 2008: 135-137). La sua trilogia sociale La Rovina (II Trono, L'Altare, L'Oro), pubblicata a Zara nel 1902, e il dramma in un atto Senza perdono, uscito sulla Rivista dalmatica (1903), furono recitate con successo in vari teatri d'Italia ricevendo le lodi da illustri letterati europei. Nel volume dedicato a Mussafia, Wondrich si presenta con la novella Ritorno alla vita (WONDRICH 1904: 79-95). Il tema sono le sofferenze e la liberazione di un giovane prete, padre Antonio, un oratore molto abile che attira il popolo con le sue prediche, che però non è capace di resistere all'assalto del dubbio e delle reminiscenze della vita civile. Tutto ciò, assieme alle provocazioni continue di un altro prete (geloso del carisma del giovane collega) lo trascina nel vortice della crisi religiosa che si risolve nella fuga dopo la visione del diavolo in veste dell'anziano collega:

Allora si vide una cosa meravigliosa. La piccola figura di Padre Anselmo scivolò tra i sai indi il nero muro, che circondava l'altare, si mosse. Una voce sepolcrale intuonò il Miserere e le volte della chiesa rimbombarono del cupo salmo cantato da tutti i frati e furono strozzate le parole del ribelle. Ed il nero muro si trasformò in un immane serpente: penetrò - fendendola in due - nella turba dei fedeli, che si ritiravano, compresi da spavento e ribrezzo, e serpeggiando si diresse al pergamo e lo salì.

La testa del mostro - Padre Anselmo - era di faccia al ribelle e scagliò su di lui la biforcuta lingua - le mani - e l'avvolse d'un soffio avvelenato - una maledizione.

[...] Ma quando i suoi piedi toccarono il suolo della chiesa, ma quando padre Anselmo stese di nuovo le mani per afferrare la preda e ricondurla nella tomba del claustro, l'ultima energia, l'ultimo soffio di vita avvivò il ribelle: egli si liberò con uno sforzo sovrumano dall'immonda spira, che lo soffocava e con voce possente, che fece fremere i fedeli e tacere la bufera, gridò il terribile 'Vade retro, Satanas'. [...] Allora il suo sguardo di fiamma si posò ancora una volta sull'altare, alla cui ombra invano aveva cercato la pace e dove aveva trovato soltanto la menzogna. [...] E Padre Antonio ritornava alla Vita: essa gli sorrise dolcemente dal cielo sereno e stellato, dal profondo e vastissimo mare, dalla madre Terra, alitante un soave profumo di rose e d'acacie, che lo accarezzò come un bacio.

(WONDRICH, 1904: 94-96)

Con questa novella il Wondrich si presenta come un autore moderno, seguendo le tendenze della letteratura italiana ed europea del tempo. II suo protagonista è un tipico personaggio decadente, in cui si manifestano i sentimenti di crisi, dubbio, in contrasto con forte devozione ed estasi religiosa, intrecciate con elementi fantastici e surreali.

Il primo dei quattro saggi degli autori zaratini dedicati a Mussafia intitolato Le lettere in Zara nel primo rinascimento, appartiene all'omonimo studio di Lorenzo Benevenia (1849-1914), distinto professore di storia, fondatore e redattore di diversi giornali zaratini nella seconda metà dell'800 (La Palestra, Annuario dalmatico, II Dalmata, 
Scintille, Rivista dalmatica). La sua produzione letteraria si ricava dai manoscritti di poesie e novelle trovati dopo la sua morte (BALIĆ-NIžlć 2008: 152). Oltre al componimento poetico più famoso, Inno di Zara, pubblica alcune poesie nella raccolta Per la Lega. All'inizio del '900 lavora a Trieste, dove scrive questo interessante studio (BENEVENIA 1904: 97-124), in cui cerca di illustrare la modesta vita culturale e letteraria della capitale dalmata le cui tracce si trovano in varie fonti archivistiche, in particolare nei testamenti e inventari delle famiglie nobili. Legando, come gli altri storici che trattarono lo stesso argomento, la nascita dell'attività letteraria allo sviluppo della lingua volgare, trascrive diversi documenti, anche ufficiali, in cui è evidente l'uso sia di espressioni in volgare (veneto), sia di quelle slave. Analizzando i manoscritti trova inoltre le differenze tra I'idioma dei patrizi e quello della plebe, e altri elementi del complesso quadro linguisticoidiomatico a Zara che si rispecchia nelle fonti scritte di quel periodo (latino, latino volgare o della plebe, idioma veneto, parole schiavizzate).

Forse l'allievo zaratino più vicino ad Adolfo Mussafia, per la carriera di docente di lingua italiana a Vienna, per l'atteggiamento e una specie di ribellione passiva contro le autorità austriache, fu Edgardo Maddalena (Zara 1867 - Firenze 1929), noto soprattutto come il più grande "goldonofilo" del suo tempo, con la più ricca raccolta di materiale legato all'opera e alla vita del sommo commediografo veneziano (BALIĆ-NIžıć 1997; 2008: 97-105). In onore del grande professore con cui strinse anche rapporti di amicizia, nel volume menzionato degli Atti pubblicati in Germania (RICHTER et al. 1905) scrive il breve saggio petrarchesco Per il bagno di Laura (MADDALENA 1905: 715-716), mentre nel volume degli studenti dalmati pubblica l'interessante studio intitolato Lazzo (MAdDALENA 1904: 125-135), in cui rielabora varie interpretazioni dell'etimologia e dell'origine del termine che

[...] significava dapprima controscena, azione giocosa, nient'altro, e più tardi nel significato del motto buffonesco venne a far parte del patrimonio della nostra lingua, ebbe frasi intiere e derivati e passò ancora nei dialetti, né la Crusca ebbe ritegno d'accoglierla nel suo santuario. I comici dell'arte la recarono in Francia e in Germania e la voce forestiera penetrò in quelle lingue.

(MADDAlena 1904: 128)

Maddalena riporta anche l'interpretazione dell'etimologia del termine in esame offerta da Luigi Riccoboni nella sua Histoire du Théâtre italien (RICCOBONI 1728):

[...] che quando Arlecchino si trova col suo padrone, occupato in faccende serie, s'egli con le sue celie interrompe il suo discorso e l'azione, taglia il corso della scena e strappa, per così dire, il filo d'un lavoro cominciato dal suo padrone. Bisogna dunque prima o poi riprendere l'argomento della scena interrotta. Conviene cioè che gli scherzi d'Arlecchino estranei al soggetto, riannodino l'azione così da parere che facciano parte dell'argomento incominciato e che ora si riprende. È perciò che questi scherzi si chiamano Lazzi o Lacci.

(MAdDALENA 1904: 129)
Lo studioso zaratino ripercorre le altre interpretazioni offerte da diversi studiosi italiani e stranieri (tra cui anche quella che il termine provenga dalla parola lax /lasci/, oppure lazo spagnolo da laqueus latino), dimostrandosi più incline ad accettare quella elaborata nella Storia del comico grottesco di Carlo Floegl, professore di filosofia a Lipsia (FLOEGL 1788: 53) alla quale si "allaccia" anche quella del Tommaseo. Secondo Floegl, nella traduzione italiana del Maddalena, "è più verosimile, che lazzi sia una forma storpiata della parola l'azione; il che trova conferma nel fatto che in vecchi scenari la parola si trova scritta spesso con una $z$, come nota lo stesso Riccoboni." (MADDALENA 1904: 132). Tommaseo, che è "nelle discipline glottologiche tutt'altro che infedelis!" riprende questa interpretazione e nel suo Dizionario della lingua italiana, e con la sua mente, per dirla con Maddalena, "sì spesso divinatrice", nota: "Rammenta lezio, e anche atto, actio. Lazzi e si fanno e si dicono." (TOMMASEO - BeluINI 1869: 1780). L'ultimo a riprendere la questione, riporta Maddalena, fu Antonio Valeri, acuto ricercatore di documenti. Secondo le sue ricerche, sembra che la parola lazzo, nel 1611 non esistesse, e invece si usasse l'espressione: far scena o attione ridicolosa (MADDALENA 1904: 133) Dopo la presentazione di alcune altre ricorrenze del termine lo studioso zaratino conclude: "Ecco dunque già formata la parola lazzo risultante dalla fusione dell'articolo / con la voce monca azzo." Fa riferimenti anche al problema di come viene scritta e pronunciata la parola, perché, se si accetta l'origine proposta da Floegl e Valeri, dovrebbe avere la $z$ sorda. La pronuncia tenue della $z$ si potrebbe spiegare con l'esser nata forse la parola "in bocca lombarda, avvezza a terminazioni in az con la $z$ sonora". Maddalena chiude l'interessante rassegna delle interpretazioni etimologiche del termine lazzo con le seguenti parole: "Comunque sia - lasciamo ai glottologi vincere questo non insormontabile ostacolo (della pronuncia, n.dell'a.) - la proposta del Floegl, così efficacemente appoggiata dal Valeri, mi sembra degna d'esser presa in seria considerazione, e forse per essa resta definitivamente fissato l'etimo della parola lazzo." (MAdDALENA 1904: 135).

Un omaggio ad Adolfo Mussafia lo rende anche Giuseppe Sabalich (1856-1928), storico, poligrafo e il più fecondo scrittore zaratino a cavallo tra i secoli XIX e XX (BALIĆNižlć 2008: 25-63). Del suo voluminoso opus in questo volume si pubblica lo studio Tradizioni popolari zaratine (SABALICH 1904: 155-174) risultato del suo interesse per "la sacra tradizione della sapienza popolare" (SABALICH 1904: 157). Nell'ambito della ricerca fatta tra vecchi zaratini e le donne che con le abitudini casalinghe conservano la summenzionata tradizione della sapienza popolare, Sabalich ha tratto dall'uso vivo un gruppo di proverbi e sentenze che "ritraggono curiose varianti e piccoli tesori di festività dialettale nell'arguzie plebea". Da questa raccolta del vastissimo materiale, che considera come un prontuario, riporta alcuni esempi, allo scopo di suscitare l'interesse di altri studiosi di estendere le loro ricerche sulla grande affinità che il dialetto, cioè i vernacolo zaratino ha con gli altri vernacoli d'Italia. Facendo riferimento al suo Saggio di voci, modi e proverbi nella parlata popolare zaratina (SABALICH 1892) e all'opera Tradizion 
popolari albonesi di Tomaso Luciani (LUCIANI 1892), Sabalich trova somiglianze tra il vernacolo zaratino ed albonese, ad esempio: l'ha bevù l'aqua de la cisterna, no l'va più via, in dialetto albonese, trova un perfetto riscontro di significato nello zaratino l'à bevudo l'acqua dei çinque pozzi. L'autore poi offre alcuni esempi di proverbi toscani e di altre regioni del bacino adriatico, cercando di scoprire un'analogia con il vernacolo zaratino. Cita inoltre alcune canzoncine, cantilene e filastrocche, italo-venete, usate in Istria e a Zara, ad esempio: "Din don, din don,/ le campane de san Simon,/ le sonava tanto forte/ le butava zo le porte", che assomiglia alla canzoncina albonese: "Din den don,/ quatro pute sul porton, ecc." (SABALICH 1904: 171). Alla fine conclude con rammarico che i proverbi e le tradizioni popolari in genere stanno scomparendo rapidamente, perché

le forme nazionali, generali, unitarie, usurpano la parlata pratica colla dottrineria e invadono il campo sereno dei dialetti, onde anche il nostro vecchio dialetto coi suoi bei vezzi di pronuncia si leviga nelle forme culte, per cui fra pochi lustri esso non rimarrà che un monumento fossile di una frale più aggraziate locuzioni che attestano la potenza serena di Venezia repubblica.

(SABALICH 1904: 174)

Gaetano Feoli (Soresina 1856 - Zara 1932), noto giornalista e scrittore, fondatore e direttore, tra l'altro, di numerosi periodici dalmati del tempo quali L'Avvenire, La Difesa, II Dalmata, La voce dalmatica, Corriere della Dalmazia e II Littorio dalmatico (TACCONI 1932: 147), pubblica un interessante saggio intitolato Un episodio poco noto di storia italiana (FEOLI 1904: 175-221) sulla deportazione in Dalmazia di un gruppo di cisalpini, imprigionati dalle autorità austriache nella fortezza di San Niccolò, che protegge le "bocche sinuose del porto di Sebenico." (FEOLI 1904: 177). L'autore spiega il contesto storico in cui accade la deportazione, descrivendo dettagliatamente le misere condizioni in cui centotrentun prigionieri politici, "patrioti repubblicani", incatenati a cinque e cinque furono cacciati come una "mandra in una nave, capace di contenerne, tutto al più, sessanta" (FEOLI 1904: 179). Dopo il viaggio faticoso, e dopo la delusione di non esser sbarcati a Zara, i captivi arrivarono al forte San Niccolò. Per illustrare le tristi condizioni in cui trascorsero alcuni mesi, Feoli cita i versi scritti posteriormente da uno dei prigionieri, il poeta mantovano Ferdinando Arrivabene. Sono versi che furono assai popolari in Italia e in Dalmazia, in particolare a Sebenico nella prima metà dell' 800 , soprattutto quelli che si riferiscono al momento in cui gli imprigionati all'arrivo si accorgono del Leone di San Marco alla porta: “Al vederci il Leone dell'Adria/ Dall'orrore un ruggito mando." (FEOLI 1904: 180).

Feoli riporta anche altre citazioni che aiutano a creare l'immagine del cupo ambiente, dove, i prigionieri, preparando nei primi giorni la terra che gli serviva da letto, trovavano anche le ossa: "Si ravvisano d'ossa insepolte/ Freddi avanzi qua e là biancheggiar" (FEOLI 1904: 181), oppure i versi che descrivono il modo in cui vennero trattati gli infelici, "precipitati dal sogno radioso della repubblica italica": "Là rinchiusi qual belve feroci/ Riservate per barbari ludi..." (FEOLI 1904: 182). Dice Feoli

\section{dell'Arrivabene e di altri prigionieri:}

Ferdinando Arrivabene, mantovano, canta le pene sofferte nella fortezza, e non pensa che un giorno - legislatore e poeta - avrà I'amicizia di Ugo Foscolo, il fanciullo cresciuto sovra una spiaggia vicina. Altri scrivono altre poesie e compongono girolette. Potrebbero - con un colpo di mano - tentare un'evasione: pochi i venti soldati contro i moltissimi giovani, forti e animosi. Ma è periglioso il far evadere dei vecchi e dei convalescenti, e per essi, l'impresa potrebbe fallire.

(FEOLI 1904: 183)

In seguito l'autore spiega come finalmente sono migliorate le condizioni per gli incarcerati grazie ai rapporti di amicizia stabiliti con la gente locale, che pure li consolava informandoli della possibilità di un trasloco, dello scambio, forse, con altri prigionieri, persino della liberazione. Descrive infine anche una sua visita dopo tanti anni al forte di S. Niccolò, in cerca delle tracce della presenza di cisalpini, e la delusione di non aver trovato niente:

lo cercavo le ossa insepolte, un anello, un solo anello, delle orrende catene; un ricordo, infinitesimo ma ancor palpitante, dell'episodio che datava giusto da un secolo. Nulla, nulla, nulla! Lungo i corridoi, su per le scale e persino sulla piazza del magnifico forte veneto, ridotta a giardino, la rigidezza militare, allineata e prosaica, aveva fatti passare i suoi badili, le sue scope, i suoi pennelli, uniformando tutto, nell'ombra del sotterraneo e su, nella gran gioia del sole. Era cancellata la breve epopea giacobina. Nuove feste, forse, si potrebbero bandire nelle candide casamatte, già confidenti alle lacrime, alle speranze, ai trastulli, agli amori di tanti patrioti italiani.

(FEOLI 1904: 189)

Nell'ultima parte dello studio, a base dei documenti ufficiali conservati nell'archivio della luogotenenza dalmata, Feoli cerca di dare una versione "ufficiale" di come erano andate le cose colla deportazione, mettendo alla luce il ruolo del conte Giambattista Stratico, consigliere al governo di Zara, nei tentativi di far finire il martirio di quegli umili ed ignoti patrioti, e trasportarli in qualche altro luogo con condizioni migliori. Narra la storia anche di altri quattro gruppi portati al forte (erano complessivamente cinque), concludendo: "E qui non so neanche dire se gli ultimi prigionieri abbian trovato l'ultimo carcere sotto le tavole di quel palcoscenico, sul quale Niccolò Tommaseo, fanciullo, doveva veder recitate più tardi le commedie di Beaumarchais, perché il documento, qui, s'arresta." (FEol 1904: 216). Alla fine del saggio, Feoli riporta un supplemento prezioso, I'“Indicazione dei 131 detenuti nel Castello di San Niccolò in Sebenico", copia dell'elenco inviato il 21 giugno 1800 dall'i.r. governo di Venezia a quello di Zara, contenente nome, cognome, età, professione e luogo di dimora dei prigionieri. 
In conclusione si potrebbe dire che i contributi analizzati di autori zaratini scritti in onore di Adolfo Mussafia, rispecchiano diversi interessi, studi e discipline presenti nell'intero volume. Come si è potuto vedere, si tratta di testi letterari, studi di storia, storia letteraria, linguistica e filologia, tutti accomunati dal comune desiderio dei loro autori di rendere omaggio all'illustre professore. Se si prende in considerazione il momento storico in cui nasce il volume, nei primi anni del Novecento, in pieno delle lotte politiche tra gli italiani e slavi della Dalmazia nonché della lotta degli studenti italiani per la fondazione di un'Università italiana a Trieste, risulta logico lo sfondo ideologico di questa pubblicazione con la quale si vuole dare appoggio alla parte italiana. Ciò nonostante, per l'alto valore non solo storico-letterario ma anche scientifico dei contributi, e per il suo carattere interdisciplinare, il volume rimane un prezioso documento e un segno di grande stima che gli studiosi dalmati avevano per il loro corregionale, il prof. Adolfo Mussafia, che, secondo Paolo Mazzoleni (MAZzolenI 1904: 24), a suo tempo veniva annoverato tra i più autorevoli dalmati del tempo e considerato come successore del grande Niccolò Tommaseo.

\section{Adolfo Mussafia e i suoi allievi zaratini}

\section{Riassunto}

Nell'intervento sono presentati i lavori di alcuni rinomati scrittori e studiosi zaratini in lingua italiana a cavallo tra l' 800 e il ' 900 che studiarono a Vienna sotto la guida del prof. Adolfo Mussafia (Spalato 1835 - Firenze 1905), dedicandogli nel 1904, in occasione del centesimo semestre di insegnamento, un prezioso volume di opere letterarie, studi e saggi, compilato assieme ad altri allievi dalmati (Ad Adolfo Mussafia gli studenti italiani della Dalmazia MDCCCLV-MCMIV, Spalato 1904).

Nella prima parte si forniscono alcuni dati biografici su Adolfo Mussafia, basati sulle ricerche di uno dei più rinomati romanisti croati Žarko Muljačić, che in uno dei suoi studi chiarisce i dubbi sulla data di nascita del suo illustre concittadino. Vengono accentuate le curiosità riguardanti gli studi e la carriera universitaria del Mussafia, con particolare riguardo alla stima che godeva tra i colleghi ed allievi che per il suo settantesimo compleanno gli regalarono un altro volume di studi e saggi scritti in suo onore (Bausteine zur Romanische Philologie für Adolfo Mussafia zum 15. Februar 1905, Halle a.d.S.1905).

Nella seconda parte, tra i dodici contributi del volume Ad Adolfo Mussafia gli studenti italiani della Dalmazia MDCCCLV-MCMIV vengono scelti tre testi letterari e quattro saggi scritti dagli allievi zaratini del Mussafia: Antonio Cippico, Giuseppe Fabbrovich, Giorgio Wondrich, Lorenzo Benevenia, Edgadro Maddalena, Giuseppe Sabalich e Gaetano Feoli, che terminati gli studi ottennero notevole successo nel campo del giornalismo, della letteratura, della storia e della filologia. I loro contributi vengono analizzati e presentati come specchio di carattere multidisciplinare del volume e dell'influsso che ebbe sulle loro carriere il Mussafia, apprezzato anche dal grande
Tommaseo e considerato nei circoli di filologi italiani come il suo successore. In conclusione viene accentuata l'importanza del volume non solo come omaggio al rinomato professore e filologo, ma anche come un documento interessante dal punto di vista culturologico e fonte di preziosi dati sull'attività letteraria e scientifica degli intellettuali dalmati a cavallo tra l' 800 e il ' 900.

Parole chiave: Adolfo Mussafia, scrittori zaratini in lingua italiana, relazioni letterarie italo-croate

\section{Adolfo Mussafia i njegovi zadarski student}

\section{Sažetak}

U radu su predstavljeni prilozi zadarskih pisaca i intelektualaca talijanskog jezičnog izraza s kraja 19. i početka 20. stoljeća koji su studirali u Beču i kojima je profesor bio Adolfo Mussafia (Split 1835. - Firenze 1905.), prvi redoviti profesor romanske filologije na Bečkom sveučilištu, objavljeni u prigodnom književno-znanstvenom zborniku koji su, uz ostale studente iz Dalmacije, 1904. godine posvetili Mussafiji u povodu održanog stotog semestra nastave (Ad Adolfo Mussafia gli studenti italiani della Dalmazia MDCCCLV-MCMIV, Spalato 1904).

U prvom dijelu rada iznose se biografski podaci o Adolfu Mussafiji, prvenstveno na temelju znanstvenih istraživanja jednog od najeminentnijih hrvatskih romanista Žarka Muljačića, koji u svojim radovima, između ostalog, rješava nedoumice o datumu rođenja slavnog sugrađanina. Ističu se zanimljivosti vezane uz Mussafijino obrazovanje i sveučilišnu karijeru, s posebnim osvrtom na ugled koji je uživao među kolegama i studentima i počast koju su mu odali za sedamdeseti rođendan objavljivanjem još jednog zbornika radova (Bausteine zur Romanische Philologie für Adolfo Mussafia zum 15. Februar 1905, Halle a.d.S.1905).

$\mathrm{U}$ drugom dijelu rada, od dvanaest priloga objavljenih u zborniku Ad Adolfo Mussafia gli studenti italiani della Dalmazia MDCCCLV-MCMIV prikazuju se tri književna teksta i četiri znanstvena rada koje potpisuju Mussafijini bivši zadarski studenti: Antonio Cippico, Giuseppe Fabbrovich, Giorgio Wondrich, Lorenzo Benevenia, Edgadro Maddalena, Giuseppe Sabalich i Gaetano Feoli, u to vrijeme već istaknuti književnici, povjesničari, filolozi i publicisti. Njihovi prilozi odraz su interdisciplinarnog karaktera zbornika i utjecaja koji je na formiranje njihovih književnih i znanstvenih interesa imao Mussafia, svojevremeno vrlo cijenjen od strane Tommasea i smatran, među talijanskim filolozima, njegovim nasljednikom. Ističe se na kraju važnost zbornika kao hommagea priznatom profesoru i filologu, ali i kao zanimljivog dokumenta s kulturološkog stajališta te kao vrijednog izvora činjenica o književnoj i znanstvenoj djelatnosti dalmatinskih intelektualaca talijanskog jezičnog izričaja na prijelazu iz 19. u 20. stoljeće.

Ključne riječi: Adolfo Mussafia, zadarski pisci talijanskog jezičnog izričaja, hrvatsko-talijanske književne veze 


\section{BIBLIOGRAFIA:}

BALIĆ-NIŽıć 1997

Nedjeljka Balić-Nižić: „Europski dometi književnog istraživanja Zadranina Edgarda Maddalene", Radovi Filozofskog fakulteta u Zadru, Razdio filoloških znanosti, 34-35 (24-25), 1995-1996, Zadar 1997., 211-222.

BALIĆ-NIŽIĆ 1998

Nedjeljka Balić-Nižić: Talijanski pisci u Zadru pred prvi svjetski rat (1900.-1915.), Rijeka - Fiume 1998.

BALIĆ-NIŽı́ć 2008

Nedjeljka Balić-Nižić: Scrittori italiani a Zara negli anni precedenti la prima guerra mondiale (1900-1915), Rita Tolomeo (a cura di), trad. Zdravka Krpina, Roma 2008.

BENEVENIA 1904

Lorenzo Benevenia: "Le lettere in Zara nel primo rinascimento", in Ad Adolfo Mussafia gli studenti italiani della Dalmazia. MDCCCLV-MCMIV, Spalato 1904, 97-124

CIPPICO 1904

Antonio Cippico: "Alla Dalmazia", in Ad Adolfo Mussafia gli studenti italiani della Dalmazia. MDCCCLV-MCMIV, Spalato 1904, 45-47

FABBROVICH 1904

Giuseppe Fabbrovich: "Vittime (Frammenti d'un romanzo... allo studio)", in Ad Adolfo Mussafia gli studenti italiani della Dalmazia. MDCCCLV-MCMIV, Spalato 1904, 49-77.

FEOLI 1904

Gaetano Feoli: "Un episodio poco noto di storia italiana", in Ad Adolfo Mussafia gli studenti italiani della Dalmazia. MDCCCLV-MCMIV, Spalato 1904, 175-221.

FLOEGL 1788

Carlo Floegl: Gescichte des Groteskekomischen, ein Beitrag zur Geschichte der Menschheit, Leipzig 1788

LUCIANI 1892

Tomaso Luciani: Tradizioni popolari albonesi, Capodistria 1892.

MADDALENA 1904

Edgardo Maddalena: "Lazzo", in Ad Adolfo Mussafia gli studenti italiani della Dalmazia. MDCCCLV-MCMIV, Spalato 1904, 125-135.

MADDALENA 1905

Edgardo Maddalena: "Per il bagno di Laura", in Bausteine zur Romanische Philologie für Adolfo Mussafia zum 15. Februar 1905, Halle a.d.S., 1905, 715-716.

MAZZOLENI 1904

Paolo Mazzoleni, "Agli studenti italiani della Dalmazia", in Ad Adolfo Mussafia gli studenti italiani della Dalmazia. MDCCCLV-MCMIV, Spalato 1904, 21-41.

\section{MULAČı́́ 1997}

Žarko Muljačić: “Novi podaci o Splićaninu Adolfu Mussafiji, prvom redovitom profesoru romanistike u Beču", Građa i prilozi za povijest Dalmacije, 13, Split 1997 301-324.

MULAČıć 2002

Žarko Muljačić: "Uno pseudo enigma concernente la biografia di Adolfo Mussafia, primo ordinario di filologia romanza all'Università di Vienna", Estudis Romànics, XXIV, Barcelona 2002, 211-215.

MUSSAFIA 1860

Adolfo Mussafia: Sprachlehre in Regeln und Beispielen, für den ersten Unterricht bearbeitet, Wien 1860.

RICCOBONI 1728

Luigi Riccoboni: Histoire du Théâtre italien, Paris 1728.

RICHTER et al. 1905

Elise Richter et al.: Bausteine zur Romanische Philologie für Adolfo Mussafia zum 15. Februar 1905, Halle a.d.S., Verlag von Max Niemeyer 1905.

SABALICH 1892

Giuseppe Sabalich: Saggio di voci, modi e proverbi nella parlata popolare zaratina Zara 1892.

SABALICH 1904

Giuseppe Sabalich: "Tradizioni popolari zaratine", in Ad Adolfo Mussafia gli studenti italiani della Dalmazia. MDCCCLV-MCMIV, Spalato 1904, 155-174.

TACCONI 1932

Ildebrando Tacconi: "Gaetano Feoli. Necrologio", Rivista dalmatica, XIII, 1-2, Zara $1932,147$.

TOLOMEO 2012

Rita Tolomeo: “Adolfo Mussafia”, in Dizionario biografico degli italiani, 77, 2012.

TOMMASEO 1872

Niccolò Tommaseo: Poesie, Firenze 1872

TOMMASEO - BELLINI 1869

Niccolò Tommaseo; Bernardo Bellini: Dizionario della lingua italiana, Torino 1869.

WONDRICH 1904

Giorgio Wondrich: "Ritorno alla vita", in Ad Adolfo Mussafia gli studenti italiani della Dalmazia. MDCCCLV-MCMIV, Spalato 1904, 79-95.

http://www.treccani.it/enciclopedia/adolfo-mussafia_(Dizionario-Biografico)

$$
\text { (31.12.2014.) }
$$

https://archive.org/details/bausteinezurrom00muss (30.12.2014.) 